\title{
Molecular epidemiology and antimicrobial resistance profiles of Salmonella isolates from dairy heifer calves and adult lactating cows in a Mediterranean pasture-based system of Australia
}

\author{
J. W. Aleri, ${ }^{1,2,3 *}$ † S. Sahibzada, ${ }^{1,3 *}$ A. Harb,,${ }^{1,3}$ A. D. Fisher,,${ }^{4,5}$ F. K. Waichigo, ${ }^{6}$ T. Lee, ${ }^{1,3}$ I. D. Robertson, ${ }^{1,7 *}$ \\ and S. Abraham ${ }^{1,3 *}$ \\ ${ }^{1}$ School of Veterinary Medicine, College of Science, Health, Engineering and Education, Murdoch University, 90 South Street, Murdoch, 6150 WA, \\ Australia \\ ${ }^{2}$ Centre for Animal Production and Health, Future Foods Institute, Murdoch University, 90 South Street, Murdoch, 6150 WA, Australia \\ ${ }^{3}$ Antimicrobial Resistance and Infectious Diseases Research Laboratory, Harry Butler Institute, Murdoch University, 90 South Street, Murdoch, \\ 6150 WA, Australia \\ ${ }^{4}$ Faculty of Veterinary and Agricultural Sciences, University of Melbourne, 250 Princes Highway, Werribee, 3030 VIC, Australia \\ ${ }^{5}$ Animal Welfare Science Centre, University of Melbourne, 3010 VIC, Australia \\ ${ }^{6}$ Brunswick Veterinary Services, 27 Ommaney Road, Brunswick Junction, 6224 WA, Australia \\ ${ }^{7}$ College of Veterinary Medicine, Huazhong Agricultural University. Wuhan, Hubei, 430070, China
}

\begin{abstract}
Dairy cows can be reservoirs of foodborne pathogens such as Salmonella that pose serious public health risks to humans. The study was designed to examine the molecular epidemiology and antimicrobial resistance profiles of Salmonella isolates from dairy heifer calves and adult lactating cows in the pasture-based system of Australia. A total of 838 animals (328 heifer calves and 510 lactating cows) from 22 farms were sampled. Overall, 54 Salmonella isolates were recovered (calves 28/328 and cows 26/510). A herd-level Salmonella prevalence of $50 \%$ (95\% confidence interval: 31\%-69\%) was recorded. Within-herd prevalence for Salmonella ranged between $4 \%-29 \%$ and $4 \%-45 \%$ among the heifer calves and adult lactating cows, respectively. Three different serovars were identified with Salmonella Infantis being the most common serovar $(\mathrm{n}=33,61 \%)$ followed by Salmonella Kiambu ( $\mathrm{n}=20,37.0 \%)$ and one isolate of Salmonella Cerro (2\%). The highest antimicrobial resistance prevalence of Salmonella isolates was found against streptomycin $(\mathrm{n}=31,57 \%)$, followed by cefoxitin $(\mathrm{n}=12,22 \%)$, ceftriaxone $(\mathrm{n}=2,4 \%)$, and chloramphenicol $(\mathrm{n}=1,2 \%)$. Multiple class resistance was observed on 4 isolates against cefoxitin, chloramphenicol, and streptomycin. Multilocus sequence types ST32 (61\%), ST309 (37\%), and ST367 (2\%) were strongly linked to the serovars Salmonella Infantis, Salmonella Kiambu, and Salmonella Cerro, respectively. Whole
\end{abstract}

Received July 29, 2021.

Accepted October 23, 2021.

*These authors contributed equally to this work.

†Corresponding author: J.Aleri@murdoch.edu.au genome sequencing of Salmonella isolates detected only 2 resistance genes: $a a c\left(6^{\prime}\right)$ gene that confers resistance against aminoglycosides among $40.7 \%$ of the isolates, and a single isolate positive for the blaDHA-16 gene. Two distinct clusters among the serovars were observed suggesting 2 independent sources of spread. Despite the low prevalence of antimicrobial resistance among Salmonella from the dairy farms, our findings contribute to the regional and national understanding of antimicrobial resistance in dairy herds in Australia. There is need for continued antimicrobial resistance stewardship and surveillance programs to ensure the production of high-quality food products and the long-term protection of both animal and human health.

Key words: prevalence, Salmonella, Salmonella Infantis, zoonoses

\section{INTRODUCTION}

Salmonellosis is a predominantly intestinal infection of humans and animals caused by numerous Salmonella enterica serovars (Barrow and Methner, 2013; Eng et al., 2015; OIE, 2019). In cattle, as with most livestock, Salmonella is carried by a proportion of animals and proliferates under conditions that are stressful to the animal, such as starvation, antimicrobial treatment, fluctuating temperature, and transport, resulting in enhanced fecal shedding in carrier animals and clinical infections such as diarrhea in a small proportion of animals (OIE, 2019). The predominant serovars detected in dairy cattle include Typhimurium, Dublin, Newport, and Infantis (OIE, 2019). In cattle, the disease is of significant public health concern due to its zoonotic nature, global distribution, wide host range, epidemiology, long carrier status in healthy animals, 
and association with increased antimicrobial resistance (AMR; Eng et al., 2015; Wang et al., 2020). Recovered animals can remain permanently infected, posing a high risk of spreading the disease. Salmonella can be disseminated within and between herds via active carriers (those individuals that are constantly shedding the bacteria), passive carriers, or latent carriers (individuals intermittently shedding the bacteria when stressed or immunosuppressed) (Holschbach and Peek, 2018). Globally there is an increased focus on Salmonella due to its zoonotic potential and increasing report of AMR to critically important antimicrobials used for treating serious infections in humans.

The use of critically important antimicrobials (CIA) such as colistin, carbapenems, extended-spectrum cephalosporins, and fluoroquinolones has been of concern due to increased AMR (Alexander et al., 2009; Davidson et al., 2018; Sobur et al., 2019). Despite the global increase in resistance to high-priority antimicrobials among Salmonella from livestock, recent studies have demonstrated AMR ecology is different and unique among Salmonella from Australian livestock (Barlow et al., 2015; Abraham et al., 2019; Sodagari et al., 2021). In a recent survey, $17 \%$ of Salmonella isolates were susceptible to all tested antimicrobials and only $8 \%$ of isolates carried resistance to the highest priority antimicrobials such as extended-spectrum cephalosporins (Abraham et al., 2019). No resistance to carbapenems, colistin, and fluoroquinolone was detected among the cattle isolates. These low rates of AMR were attributed to restrictions on the use of highest-priority CIA in livestock (apart from ceftiofur) and quarantine restrictions (ban on importation of livestock and fresh meat; Mukerji et al., 2017). However, there are limited studies specifically investigating Salmonella prevalence and AMR among dairy cattle and calves. To address this, this study aimed to establish the prevalence, molecular characterization, and AMR profiles of Salmonella isolates from dairy heifer calves and adult lactating cows in a Mediterranean (characterized by hot dry summers, mild wet winters, and variable feed supplementation throughout the year) pasture-based system of Australia. It was hypothesized that Salmonella isolated from feces of dairy cattle in a Mediterranean pasture-based production system would be fully susceptible to highest priority CIA due to extensive animal production and lack of CIA use.

\section{MATERIALS AND METHODS}

\section{Ethics Approval}

The study was conducted in accordance with the Australian Code of Practice for the Care and Use of
Animals for Scientific Purposes, with the approval of the Human Research and Animal Ethics Committees of Murdoch University, approval no. R3144/19 and 2019/047, respectively.

\section{Study Area}

The study was conducted in the southwest region of Western Australia. The region has a temperate Mediterranean climate with an annual rainfall of approximately $730 \mathrm{~mm}$. Dairy farms are predominantly located southwest of Perth (capital city of Western Australia), this area being well suited to pasture-based feeding systems.

\section{Study Design and General Data Collection}

Data were collected from April 2019 to June 2020. This was a cross-sectional study where study farms were visited once to obtain fecal samples from healthy dairy heifer calves $\leq 7 \mathrm{~d}$ old and adult lactating cows. All fecal samples were obtained directly from the rectum of each animal using a sterile glove and then immediately placed into sterile screw-capped containers, labeled, and transported at $4^{\circ} \mathrm{C}$ to the Murdoch University Antimicrobial Resistance and Infectious Diseases Laboratory for bacteriological examination, where they were processed on the day of collection.

\section{Study Populations and Study Farms}

The source population included heifer calves $\leq 7 \mathrm{~d}$ old and adult lactating dairy cows reared in the southwest Mediterranean region of Western Australia. A total of 22 farms were sampled. A convenience sample of 140 registered dairy producers was invited via email. Additional expressions of interest to the dairy producers were sent via a regional newsletter (Feed Trough) and during a regional farmer's day event. Participation was voluntary, and no incentives were provided.

\section{Selection of Study Subjects}

A 2-stage cluster sampling technique was used to select study subjects. In farms with less than 25 calves at the time of sampling, all calves were included in the study. In those farms that had more than 25 calves in the required age group, a total of 25 calves were included in the study. The 25 calves or adult cows were selected randomly. For each farm, random selection, was done by first identifying all the calves and adults that met the selection criteria and numbering them serially as 1 , 2, 3 to $\mathrm{s}$, where s was the last serial number depending on the total number of calves and adults. Then, with 
the serially numbered animals, a total of 25 random numbers representing the animals to be sampled were generated in Excel (Microsoft Corp.) spreadsheets. This was done separately for the calves and adult cows. The adult lactating animals were sampled as they entered into the milking parlor at milking. A total of 20 to 25 healthy dairy heifer calves $\leq 7 \mathrm{~d}$ old and adult lactating cows were sampled from each farm.

\section{Sample Size Estimations}

The following assumptions were made for the purposes of a sample size calculation based on our previous study (Aleri et al., 2021): that if the true proportion of farms with optimal calf rearing practices is $20 \%$, and the survey is $100 \%$ sensitive and specific in identifying optimal practice, with a sample size of $44,95 \%$ of the time, the true proportion will be with an interval of $\pm 10 \%$ of our estimated proportion (Sergeant, 2018). With a $10 \%$ inclusion for farm nonresponse rate and missing data, a total of 50 farms were targeted. In a separate scenario, a sample size for the number of calves and adults to estimate prevalence of Salmonella in healthy animals was determined, and a total of 862 animals was deemed appropriate. A 50\% prevalence of Salmonella was assumed based on diagnostic tests utilizing $85 \%$ sensitivity and $85 \%$ specificity with a $95 \%$ confidence interval while allowing for a $5 \%$ error rate; hence, the total was 784 animals before a $10 \%$ inclusion for nonresponse rate. Therefore, approximately 20 to 25 calves aged $\leq 7 \mathrm{~d}$ and adult lactating cows were sampled per farm.

\section{Isolation and Identification of Salmonella}

Salmonella isolation was performed according to the ISO 6579-1:2017 standards and procedures (Mooijman et al., 2019). Briefly, $3 \mathrm{~g}$ of each fecal sample was transferred aseptically to $8 \mathrm{~mL}$ of sterile buffered peptone water and incubated for 18 to $24 \mathrm{~h}$ at $37^{\circ} \mathrm{C}$. A $1,000-\mu \mathrm{L}$ aliquot, or $1 \mathrm{~mL}$, of pre-enriched suspension was transferred into $9 \mathrm{~mL}$ of Rappaport-Vassiliadis broth (Oxoid/Thermo Fisher Scientific), and incubated at $42^{\circ} \mathrm{C}$ for $24 \mathrm{~h}$. A loopful of broth culture was streaked on xylose lysine deoxycholate (Micromedia, Edwards) agar and Brilliance Salmonella agar (Thermo Fisher Scientific), and the plates were incubated at $37^{\circ} \mathrm{C}$ for $24 \mathrm{~h}$. Presumptive colonies with Salmonella morphology from each plate (e.g., black and purple colonies on xylose lysine deoxycholate and Brilliance Salmonella agars, respectively) were then confirmed by using MALDI-TOF analysis as per the manufacturer's recommendation (Bruker).

\section{Antimicrobial Susceptibility Testing}

Antimicrobial susceptibility testing on recovered isolates was performed by the broth microdilution MIC method using the Sensititer system and AUS1MUR Sensititer NARMS panel (Trek Diagnostics, Thermo Fisher Scientific). The MIC results were captured using Vision System (Trek Diagnostics, Thermo Fisher Scientific) and the results were interpreted and verified independently by 2 laboratory scientists. The MIC were interpreted using epidemiologic cut-off values (ECOFF) following the European Committee's guidelines on Antimicrobial Susceptibility Testing (EUCAST, 2021). The ECOFF values for 2 antibiotics, ceftriaxone and colistin, were lacking in EUCAST, and were used from Tyson et al. (2018). The isolates were tested against the following 16 different antimicrobials: amoxicillin, ampicillin, azithromycin, cefoxitin, ceftiofur, ceftriaxone, chloramphenicol, ciprofloxacin, colistin, florfenicol, gentamicin, meropenem, nalidixic acid, streptomycin, tetracycline, and trimethoprim sulfamethoxazole. Inoculation and incubation were carried out as per the manufacturer's guidelines, with quality control organism Escherichia coli ATCC25922. The isolates were classified as multi-class resistant (MCR) if they exhibited MIC above the ECOFF for one or more antimicrobial agents in 3 or more antimicrobial classes. Non-wild-type isolates (defined as having MIC above the ECOFF) have been shown to contain acquired resistance mechanism in their genome, even though they may have MIC below the defined Clinical and Laboratory Standards Institute (CLSI) clinical breakpoints. Therefore, for more simplistic determination of individual and MCR isolates, we refer to isolates exceeding the antimicrobial ECOFF as resistant.

\section{Whole-Genome Sequencing}

All Salmonella isolates underwent whole-genome sequencing using Illumina Nextera XT chemistry as previously described. Extraction of DNA was performed using the MagMAX Multi-sample DNA extraction kit (Thermo Fisher Scientific) as per the manufacturer's instructions. The genomes that passed the quality tests were used to predict the sequence type (ST) using the mlst-2.19 package (Seemann, 2019). The AMR genes and virulence genes were detected via resfinder (Zankari et al., 2012) and the universal virulence finder database (Zhang et al., 2019) using ABRicate V1.0.1 (Seemann, 2021). A cut-off value for gene coverage with $100 \%$ and identity over $95 \%$ was used. The serotyping prediction was performed on the WGS data using SeqSero2 V1.2.1 (Zhang et al., 2019). Genome sequences were annotated 
Table 1. Description of herd- and animal-level prevalence and Salmonella serovars isolated from 22 dairy farms in Western Australia

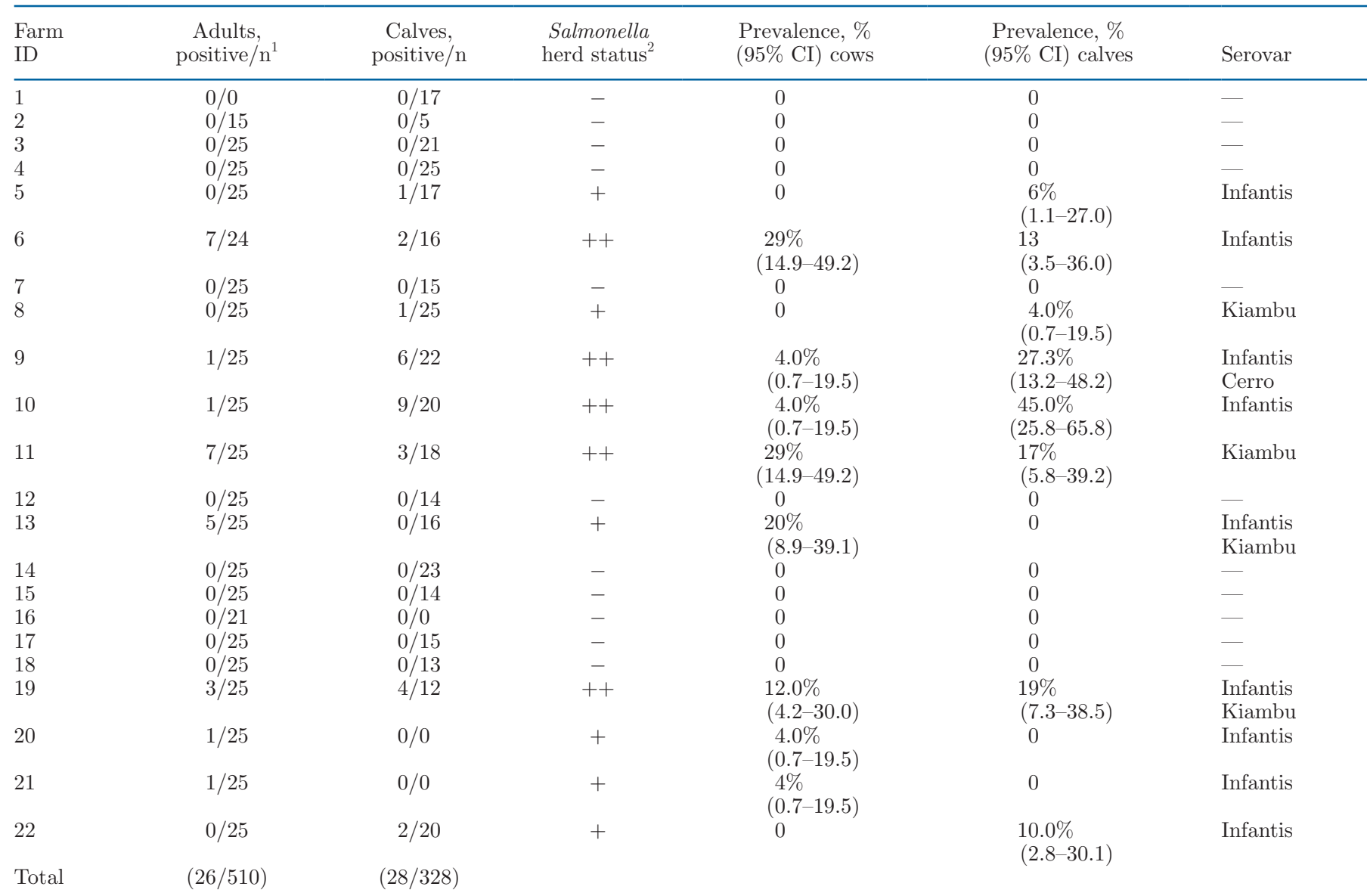

${ }^{1} \mathrm{n}=$ total number of animals sampled.

${ }^{2}+=$ positive status for Salmonella isolates for either calves or adult lactating cows; $++=$ positive status for Salmonella isolates in both calves and adult lactating cows; $-=$ negative status for Salmonella isolates in both calves and adult lactating cows.

with Prokka V1.14.6 (Seemann, 2014) and the pangenome was extracted using Roary V3.13.0 (Seemann, 2014; Page et al., 2015). Principal component analysis (PCA) of binomial variables on the pan-genome matrix was performed for determination of association for total gene content and epigenetic traits. The 95\% density ellipses were calculated (within R; R Core Team, 2021) from the specified correlation matrix (i.e., the first 2 components) and plotted with ggplot2 V3.3.3. An approximate maximum likelihood phylogenetic tree was constructed for all isolates obtained on SNP in the core genome. Core genes were aligned using SNIPPY (v4.1; Seemann, 2015).

\section{Data Analysis}

Data were analyzed using SPSS Statistics software version 22.0, 2013 (SPSS Inc.) and statistical package R (R Core Team, 2021). The frequency and 95\% confi- dence intervals of the serovars and isolates resistant to antibiotics were determined.

\section{RESULTS}

\section{General Descriptions}

A total of 838 animals from 22 farms were sampled. Of these, 328 were heifer calves and 510 adult lactating cows. A detailed description of the total number of animals sampled in each farm is shown in Table 1.

\section{Prevalence}

Of the 22 farms sampled, Salmonella was detected on 11 farms (50\%, 95\% CI: 31\%-69\%). Within-herd prevalence among the calves and adult lactating cows is detailed in Table 1. A prevalence of 36\% (95\% CI: $19 \%-57 \%$ ) was recorded for the heifer calves and adult 
lactating cows. Within-herd prevalence for Salmonella ranged between $4 \%$ to $29 \%$ and $4 \%$ to $45 \%$ among the heifer calves and adult lactating cows, respectively (Table 1). A total of 54 Salmonella isolates were recovered (calves 28/328 and cows 26/510). Three different serotypes were identified with Salmonella Infantis being the most common serovar $(\mathrm{n}=33,61 \%)$ followed by Salmonella Kiambu $(\mathrm{n}=20,37 \%)$ and one isolate of Salmonella Cerro (2\%).

\section{Phenotypic and Genotypic Characterization of Antimicrobial Resistance in Salmonella Isolates}

The 54 Salmonella isolates were screened for susceptibility to a panel of 16 antimicrobials, as presented in Figure 1. The highest frequencies of non-wild type isolates (resistant) were found against streptomycin (n $=31,57 \%)$, followed by cefoxitin $(\mathrm{n}=12,22 \%)$ and ceftriaxone ( $\mathrm{n}=2,4 \%$; Table 2). However, all isolates were susceptible to tested antimicrobials using CLSI clinical breakpoints. No clinical breakpoints are available for streptomycin. None of the isolates exhibited an MCR phenotype.

\section{Multilocus Sequence Typing of Salmonella Isolates}

In this study all serovars were represented by a single ST. Multilocus sequence typing indicated $3 \mathrm{ST}$. The majority of isolates returned as ST32 (Infantis, 61\%) and ST309 (Kiambu, 37\%), and only one isolate typed as ST367 (Cerro).

\section{Whole-Genome Sequencing of Salmonella Isolates}

The WGS analysis predicted only 2 resistance genes among all Salmonella. The $a a c\left(6^{\prime}\right)$ gene that confers resistance against aminoglycosides was found among $41 \%$ of the isolates, whereas a single isolate was positive for $b l a_{\mathrm{DHA}-16}$ gene. A total of 102 virulence genes factors were found among the tested isolates. The virulence genes were found to be associated with serovars. The isolates shared $58 \%$ of the virulence genes; however, diversification among serovars was noted for carriage of 43 different genes influencing cluster formation among the tested isolates (Figure 2). In total, 7,368 genes were identified across the 54 Salmonella isolates, whereas 3,519 genes returned as core (Supplemental Figure S1,

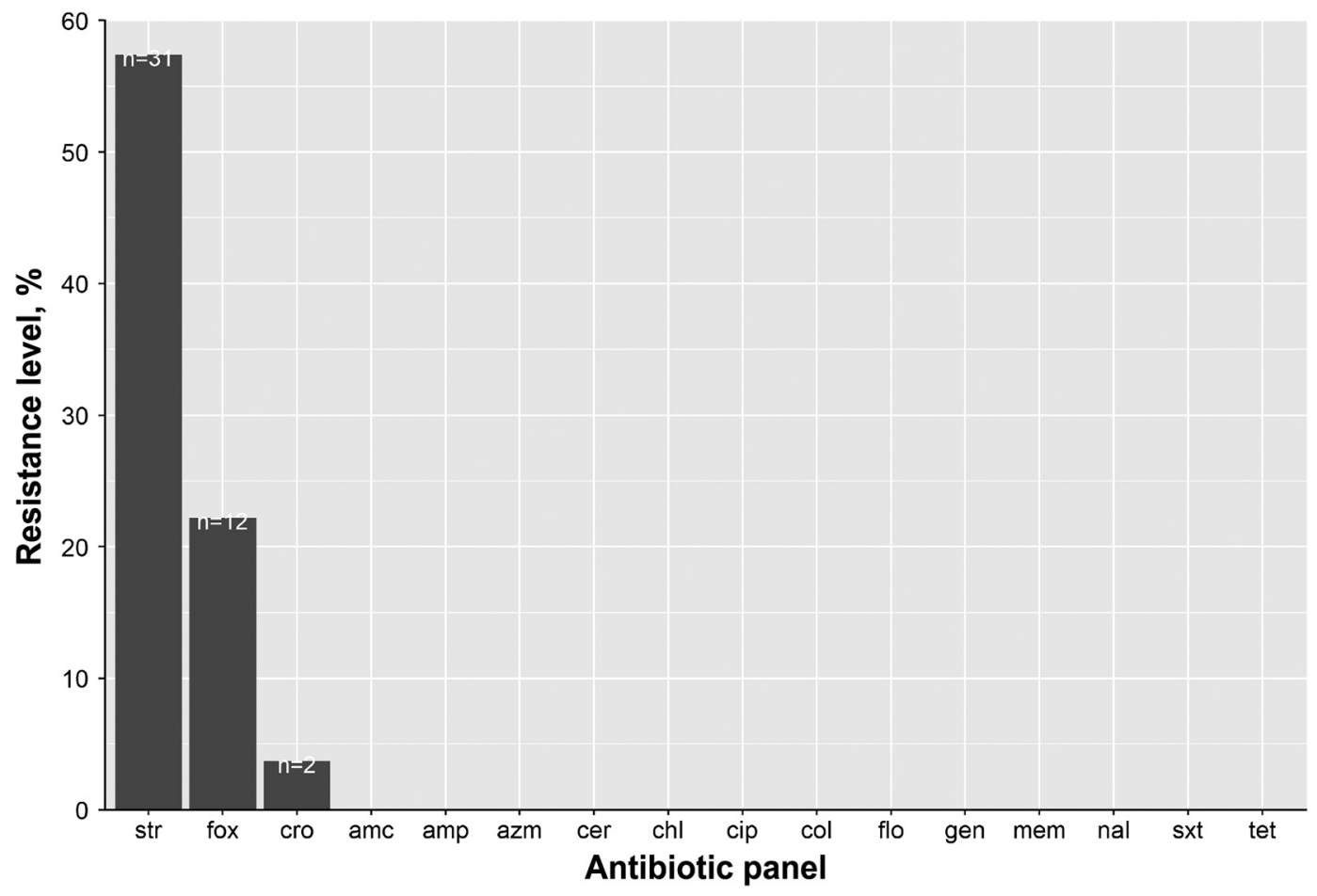

Figure 1. Proportion of wildtype isolates (resistant) Salmonella isolates. amc = amoxicillin; amp = ampicillin; azm = azithromycin; cer $=$ ceftiofur; $\mathrm{chl}=$ chloramphenicol; $\mathrm{cip}=$ ciprofloxacin; $\mathrm{col}=$ colistin; cro = ceftriaxone; flo $=$ florfenicol; fox $=$ cefoxitin; gen $=$ gentamicin; mem = meropenem; nal = nalidixic acid; str = streptomycin; sxt = trimethoprim-sulfamethoxazole; tet $=$ tetracycline. 
Table 2. Minimum inhibitory concentrations of Salmonella isolates collected from dairy farms in Western Australia ${ }^{1}$

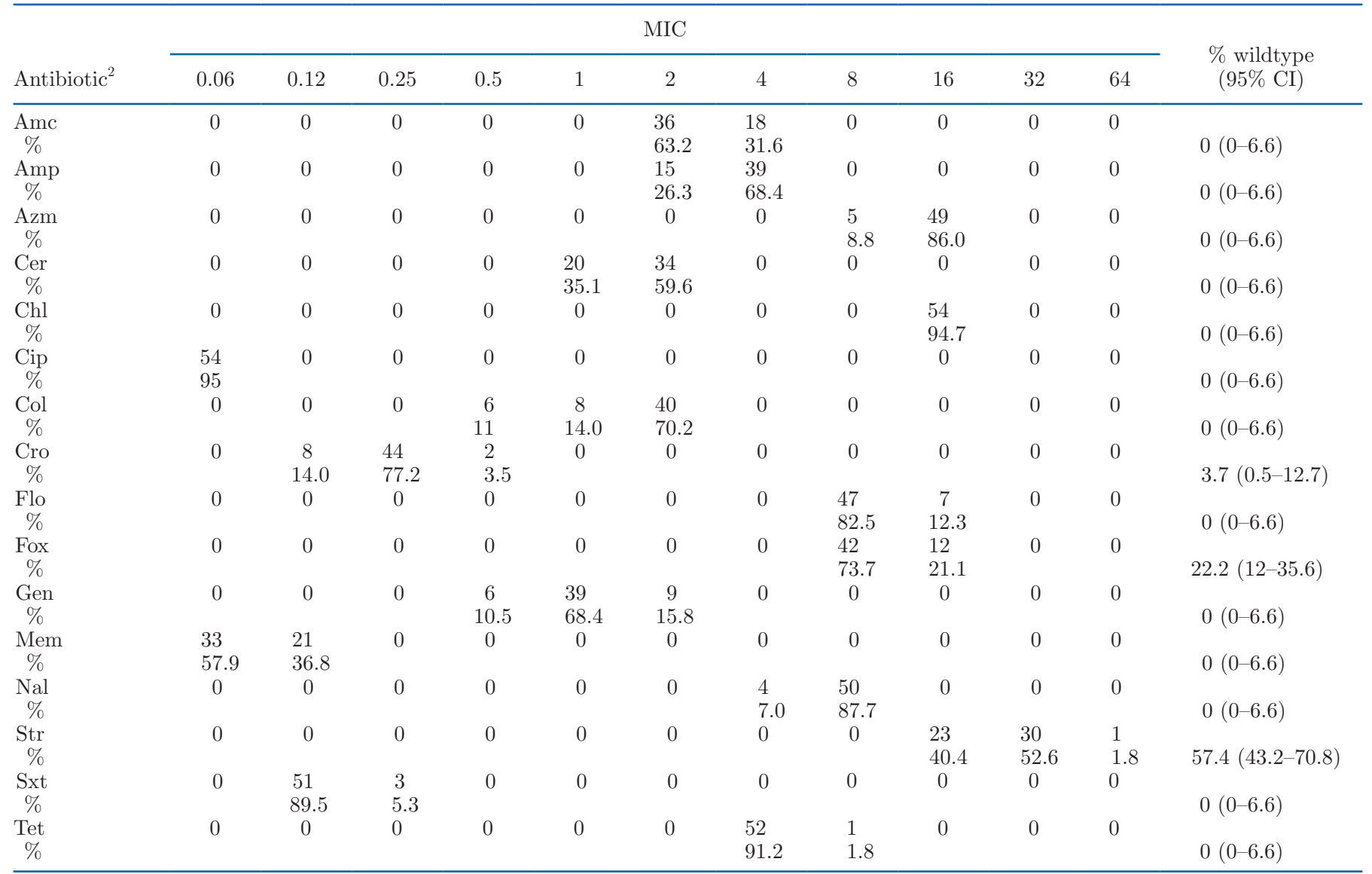

${ }^{1}$ All isolates were clinically susceptible to all antimicrobials including cefoxitin and ceftriaxone using Clinical and Laboratory Standards Institute clinical breakpoints. No clinical breakpoints were available for streptomycin.

${ }^{2} \mathrm{Amc}=$ amoxicillin; Amp = ampicillin; Azm = azithromycin; Cer = ceftiofur; Chl = chloramphenicol; Cip = ciprofloxacin; Col = colistin; Cro = ceftriaxone; Flo = florfenicol; Fox = cefoxitin; Gen = gentamicin; Mem = meropenem; Nal = nalidixic acid; Str = streptomycin; Sxt = trimethoprim sulfamethoxazole; Tet $=$ tetracycline.

https://figshare.com/articles/figure/Supplementary _information_docx/17036357/1). We found 3 distinct clusters using pan-genome as well as core genome SNP analysis. The clades were highly divergent from each other (Figure 3) and influenced by serovar carriage. Based on the presence and absence of 7,368 genes identified, principal components 1 and 2 showed 2 distinct clustering among the serovars (Figure 4). The core genome and PCA analysis demonstrated no distinct grouping within the farms.

\section{DISCUSSION}

There is an increased public health concern on the contribution of livestock production systems toward the emergence and dissemination of antimicrobialresistant Salmonella. To the authors' knowledge this is the first study investigating the prevalence of Salmo- nella in healthy heifer calves and adult lactating cows across dairy herds in Australia. The study highlights the importance of a food safety surveillance program in understanding the phenotypic and genomic characterization of Salmonella isolated from heifer calves and adult lactating cows in the southwest dairy farms of Western Australia. Overall, 54 Salmonella isolates were recovered (calves 28/328 and cows 26/510). The study showed a $50 \%(11 / 22)$ within-herd prevalence of Salmonella and with low AMR to CIA.

Salmonella in dairy farms can spread via several routes, and knowing the prevalence of Salmonella on dairy farms is valuable for devising strategies that can disrupt pathogen transmission and recolonization cycles (Callaway et al., 2005). In the present study, regardless of the age group, an overall herd-level prevalence of $50 \%$ was recorded. However, based on the age groups, lower prevalences of approximately $36 \%$ were recorded 


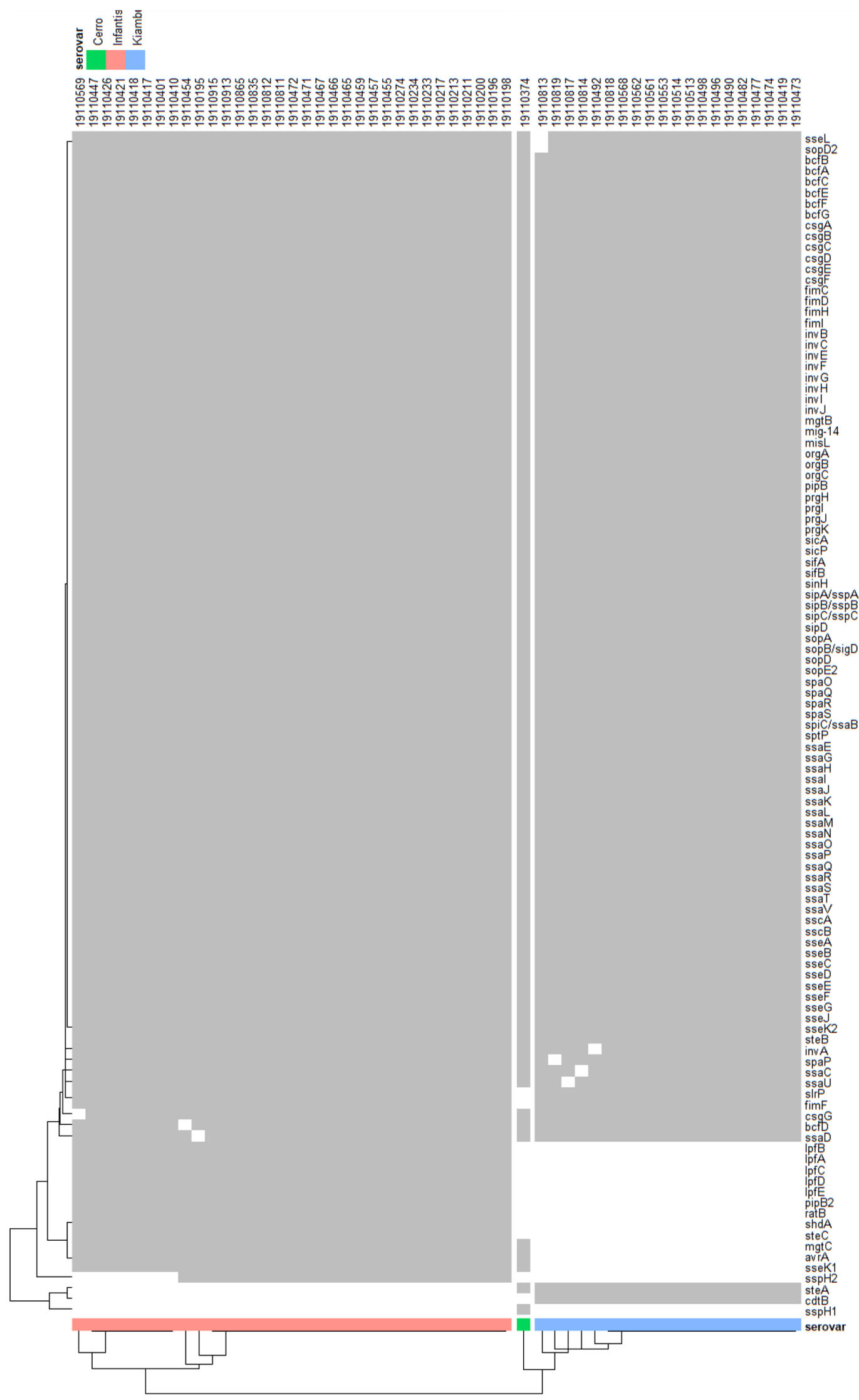

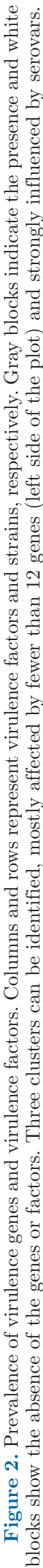


for both heifer calves and adult lactating cows. These findings are partly in agreement with the findings from an Australian study investigating the shedding patterns and prevalence of enteric pathogens in calves with diarrhea. Reports from the United States have recorded herd prevalences of 27 to $31 \%$ (Wells et al., 2001; Callaway et al., 2005). In dairy farms, animals that recover from salmonellosis can remain permanently infected, representing a high risk of spreading the disease (Holschbach and Peek, 2018). It is possible that the shedding recorded in the present study is from new subclinical cases or recovered clinical cases across the adult population.

All isolates in this study were susceptible to all tested antimicrobials using CLSI clinical breakpoints.
No clinical breakpoints were available for streptomycin; however, this drug is, along with other aminoglycosides, not clinically effective against Salmonella. Some of the isolates appeared as non-wildtypes against streptomycin and cefoxitin. These observations have not been reported in Australian dairy farms. In recent studies among Australian beef and dairy cattle, a majority (92\%) of Salmonella isolates were susceptible to all antimicrobials except florfenicol. In the same abattoir study, resistance was observed to streptomycin, ampicillin, trimethoprim sulfamethoxazole, and tetracycline among the beef cattle isolates (Barlow et al., 2015; Kidsley et al., 2018). Our present data show there was still low AMR among the Salmonella isolates. These observations would be possibly driven by lack of use

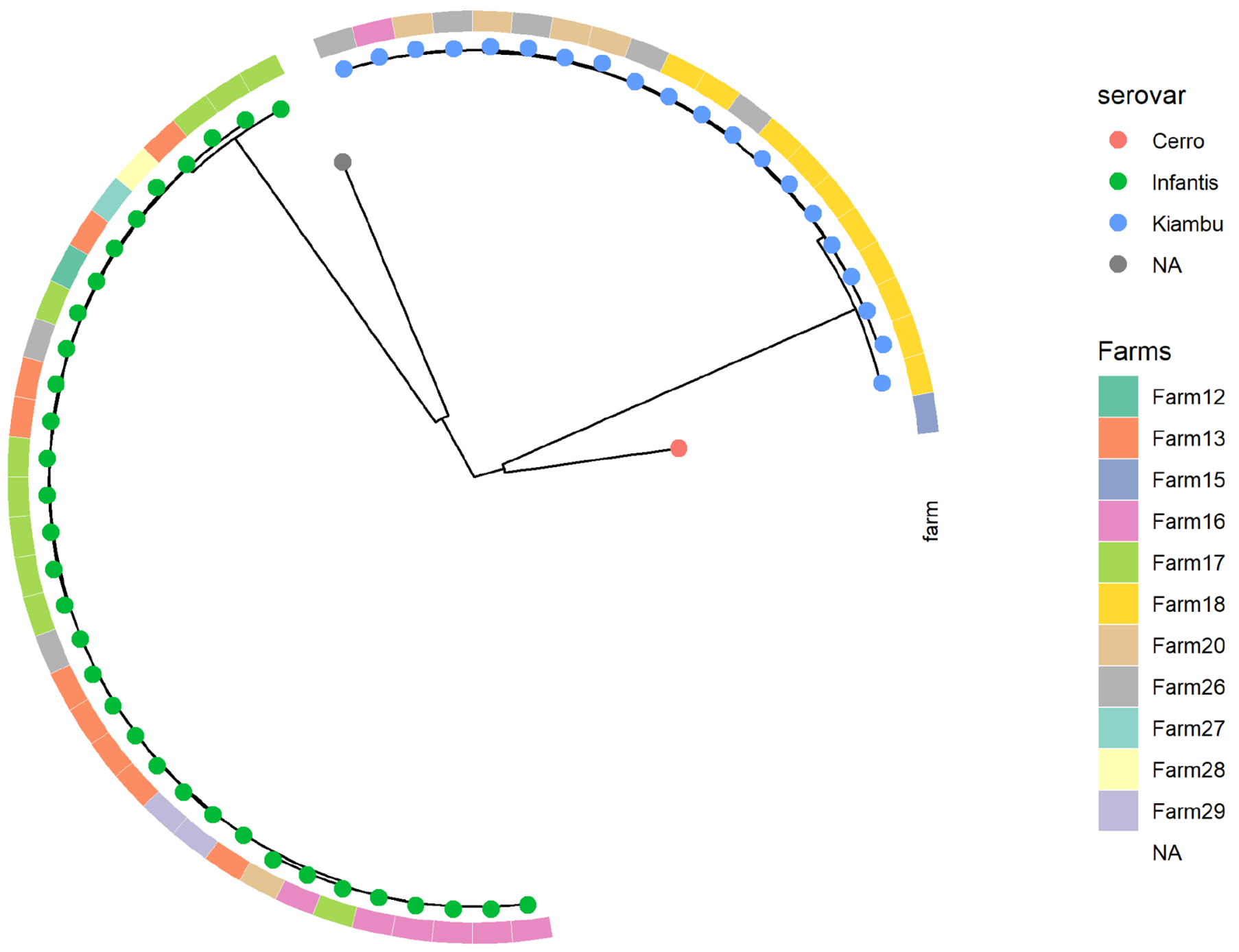

Figure 3. Maximum likelihood phylogenetic tree using core genome SNP of Salmonella isolated in dairy herds in Western Australia. There are 3 distinct clades with the top right clade consisting of cerovar Cerro (red tips), Kiambu (blue tips), and the left clade consist of serovar Infantis (green tips). NA = no serovar detected. 


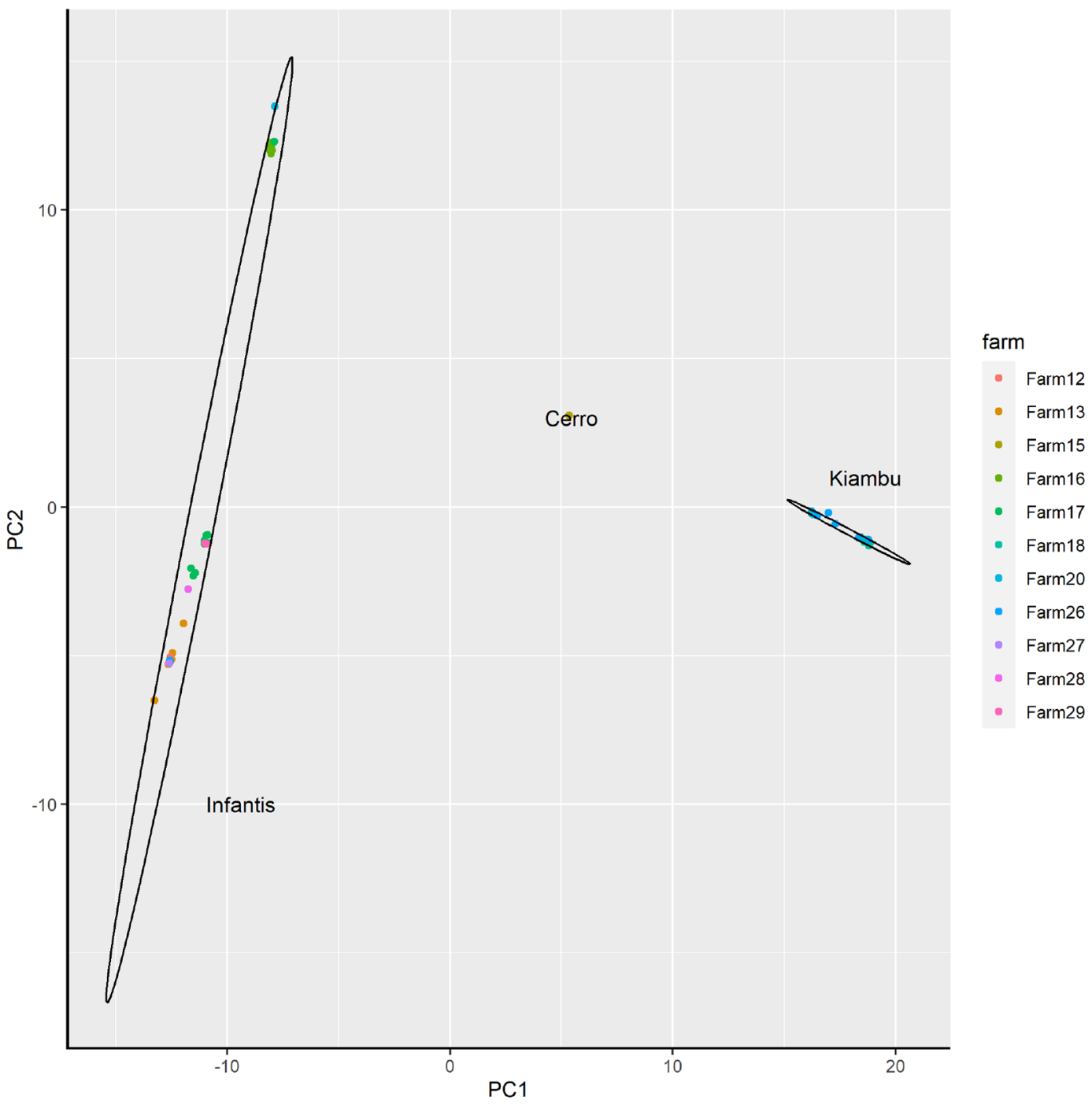

Figure 4. A principal component (PC) analysis of total gene content of Salmonella isolates from dairy cattle comprised of 3 serovars Salmonella Kiambu, Salmonella Cerro, and Salmonella Infantis. The farms are shown in different colors with a $95 \%$ density ellipse for serovars.

of CIA. There is a need to continue such antimicrobial surveillance programs across Australian dairy farms to tackle any serious AMR patterns. The findings from the present study support a hypothesis of spread among the farms. The heatmap, PCA, and SNP-based phylogenetic analysis indicate dissimilarities within the populations of Salmonella in dairy cattle. Principal component analysis based on the total gene content showed that isolates from the dairies had diverse genetic compositions highly influenced by serovar and specifically by multilocus sequence typing carriage. Similar observations were noted for the SNP-derived maximum likelihood phylogenetic tree based on the core genes. However, there was no distinct clustering observed among farms, indicating a possible transmis- sion of Salmonella between the farms. There is a need to emphasize on effective biosecurity practices among the southwest dairy farms in Western Australia.

A limitation to the study is the results provide a regional characterization of Salmonella isolates in Australia's Mediterranean pasture-based dairy production system. Future research should focus on the eastern states of Australia. There is need for longitudinal studies to understand shedding patterns among healthy carrier animals and the associated risk factors.

In our study, we found a low proportion of resistance among the Salmonella isolates and found all isolates susceptible to CIA, indicating that strict quarantine and biosecurity placed on the national border along with strict antimicrobial usage in food animals are very 
useful. Although isolates were clinically susceptible to all tested antimicrobials, some isolates returned nonwildtype against highest priority CIA such as cefoxitin and ceftriaxone. The whole-genome sequence analysis revealed no known $\beta$-lactam resistance genes imparting resistance to cefoxitin. The low MIC value among the tested Salmonella isolates to cefoxitin could potentially be due to variability in phenotypic assays in the laboratory, resulting in a phenomenon known as MIC shift (Badger et al., 2018).

\section{CONCLUSIONS}

The results provide evidence of the presence of Salmonella among dairy cows in the southwest region of Western Australia. Our findings also contribute to the regional and national understanding of AMR in dairy herds in Australia. There is a need for continued AMR surveillance programs to ensure the production of highquality food products and the long-term protection of both animal and human health.

\section{ACKNOWLEDGMENTS}

The study was supported by a grant from Dairy Australia (grant \#16085) and by Murdoch University (grant \#13363; Perth, Western Australia). We thank John Abbott, Aprille Jack, and Michele Starcevich (Murdoch University) for their technical assistance and the many producers for their kind participation in this study. Special thanks to Hamid Sodagari (Murdoch University) for the technical support. The authors have not stated any conflicts of interest.

\section{REFERENCES}

Abraham, S., M. O'Dea, S. Sahibzada, K. Hewson, A. Pavic, T. Veltman, R. Abraham, T. Harris, D. J. Trott, and D. Jordan. 2019 Escherichia coli and Salmonella spp. isolated from Australian meat chickens remain susceptible to critically important antimicrobial agents. PLoS One 14:e0224281. https://doi.org/10.1371/journal .pone.0224281.

Aleri, J. W., J. Gogoi-Tiwari, H. K. Tiwari, A. D. Fisher, F. W. Waichigo, and I. D. Robertson. 2021. Prevalence of failure of passive transfer of immunity in dairy calves in a Mediterranean pasture-based production system of the south-west region of Western Australia. Res. Vet. Sci. 139:121-126. https://doi.org/10.1016/j .rvsc.2021.07.020.

Alexander, K. A., L. D. Warnick, and M. Wiedmann. 2009. Antimicrobial resistant Salmonella in dairy cattle in the United States. Vet. Res. Commun. 33:191-209. https://doi.org/10.1007/s11259 -008-9170-7.

Badger, S., S. Abraham, S. Saputra, D. J. Trott, J. Turnidge, T. Mitchell, C. G. B. Caraguel, and D. Jordan. 2018. Relative performance of antimicrobial susceptibility assays on clinical Escherichia coli isolates from animals. Vet. Microbiol. 214:56-64. https://doi .org/10.1016/j.vetmic.2017.12.008.

Barlow, R. S., K. E. McMillan, L. L. Duffy, N. Fegan, D. Jordan, and G. E. Mellor. 2015. Prevalence and antimicrobial resistance of Sal- monella and Escherichia coli from Australian cattle populations at slaughter. J. Food Prot. 78:912-920. https://doi.org/10.4315/0362 -028X.JFP-14-476.

Barrow, P. A., and U. Methner. 2013. Salmonella in Domestic Animals. CAB International.

Callaway, T. R., J. E. Keen, T. S. Edrington, L. H. Baumgard, L. Spicer, E. S. Fonda, K. E. Griswold, T. R. Overton, M. E. VanAmburgh, R. C. Anderson, K. J. Genovese, T. L. Poole, R. B. Harvey, and D. J. Nisbet. 2005. Fecal prevalence and diversity of Salmonella species in lactating dairy cattle in four states. J. Dairy Sci. 88:3603-3608. https://doi.org/10.3168/jds.S0022-0302(05)73045 $-9$.

Davidson, K. E., B. A. Byrne, A. F. A. Pires, K. G. Magdesian, and R. V. Pereira. 2018. Antimicrobial resistance trends in fecal Salmonella isolates from northern California dairy cattle admitted to a veterinary teaching hospital, 2002-2016. PLoS One 13:e0199928. https://doi.org/10.1371/journal.pone.0199928.

Eng, S.-K., P. Pusparajah, N.-S. Ab Mutalib, H.-L. Ser, K.-G. Chan, and L.-H. Lee. 2015. Salmonella: A review on pathogenesis, epidemiology and antibiotic resistance. Front. Life Sci. 8:284-293. https: //doi.org/10.1080/21553769.2015.1051243.

EUCAST. 2021. European Committee on Antimicrobial Susceptibility Testing. Data from the EUCAST MIC distribution website. Accessed Jan. 1, 2021. http://www.eucast.org.

Holschbach, C. L., and S. F. Peek. 2018. Salmonella in dairy cattle. Vet. Clin. North Am. Food Anim. Pract. 34:133-154. https://doi .org/10.1016/j.cvfa.2017.10.005.

Kidsley, A. K., S. Abraham, J. M. Bell, M. O'Dea, T. J. Laird, D. Jordan, P. Mitchell, C. A. McDevitt, and D. J. Trott. 2018. Antimicrobial susceptibility of Escherichia coli and Salmonella spp. isolates from healthy pigs in Australia: Results of a pilot national survey. Front. Microbiol. 9:1207. https://doi.org/10.3389/fmicb .2018 .01207 .

Mooijman, K., A. Pielaat, and A. F. A. Kuijpers. 2019. Validation of EN ISO 6579-1 - Microbiology of the food chain - Horizontal method for the detection, enumeration and serotyping of Salmonella-Part 1: Detection of Salmonella spp. Int. J. Food Microbiol 288:3-12. https://doi.org/10.1016/j.ijfoodmicro.2018.03.022.

Mukerji, S., M. O'Dea, M. Barton, R. Kirkwood, T. Lee, and S. Abraham. 2017. Development and transmission of antimicrobial resistance among Gram-negative bacteria in animals and their public health impact. Essays Biochem. 61:23-35. https://doi.org/10 .1042/EBC20160055.

OIE. 2019. Manual of diagnostic tests and vaccines for terrestrial animals. OIE; 2013. Salmonellosis. Accessed Jun. 1, 2020. https:// www.oie.int/fileadmin/Home/fr/Health_standards/tahm/3.09.08 SALMONELLOSIS.pdf.

Page, A. J., C. A. Cummins, M. Hunt, V. K. Wong, S. Reuter, M. T. G. Holden, M. Fookes, D. Falush, J. A. Keane, and J. Parkhill. 2015. Roary: Rapid large-scale prokaryote pan genome analysis. Bioinformatics 31:3691-3693. https://doi.org/10.1093/bioinformatics/ btv421.

R Core Team. 2021. R: A language and environment for statistical computing [Computer software, version 4.0.5]. R Foundation for Statistical Computing. Accessed Apr. 6, 2021. http://www.R -project.org.

Seemann, T. 2014. Prokka: Rapid prokaryotic genome annotation. Bioinformatics 30:2068-2069. https://doi.org/10.1093/ bioinformatics/btu153.

Seemann, T. 2015. Snippy: Fast bacterial variant calling from NGS reads.

Seemann, T. 2019. mlst. Github (Version 2.19). Accessed Apr. 6, 2021. https://github.com/tseemann/mlst.

Seemann, T. 2021. ABRicate. GIthub (V1.0.1). Accessed Apr. 6, 2021. https://github.com/tseemann/abricate.

Sergeant, E. S. G. 2018. Epitools: Epidemiological calculators. Ausvet. Accessed Nov. 24, 2021. http://epitools.ausvet.com.au.

Sobur, M. A., A. A. M. Sabuj, R. Sarker, A. M. M. T. Rahman, S. M. L. Kabir, and M. T. Rahman. 2019. Antibiotic-resistant Escherichia coli and Salmonella spp. associated with dairy cattle and 
farm environment having public health significance. Vet. World 12:984-993. https://doi.org/10.14202/vetworld.2019.984-993.

Sodagari, H. R., S. Sahibzada, I. Robertson, I. Habib, and P. Wang. 2021. Whole-genome comparative analysis reveals association between Salmonella genomic variation and egg production systems. Front. Vet. Sci. 8:666767. https://doi.org/10.3389/fvets.2021 .666767 .

Tyson, G. H., S. Bodeis-Jones, H. Caidi, K. Cook, U. Dessai, J. Haro, A. E. McCullough, J. Meng, C. A. Morales, J. P. Lawrence, G. E. Tillman, A. Winslow, and R. A. Miller. 2018. Proposed epidemiological cutoff values for ceftriaxone, cefepime, and colistin in Salmonella. Foodborne Pathog. Dis. 15:701-704. https://doi.org/ 10.1089/fpd.2018.2490.

Wang, J., K. Xue, P. Yi, X. Zhu, Q. Peng, Z. Wang, Y. Peng, Y. Chen, I. D. Robertson, X. Li, A. Guo, and J. W. Aleri. 2020. An abattoir-based study on the prevalence of Salmonella fecal carriage and ESBL related antimicrobial resistance from culled adult dairy cows in Wuhan, China. Pathogens 9:853. https://doi.org/10.3390/ pathogens 9100853.

Wells, S. J., P. J. Fedorka-Cray, D. A. Dargatz, K. Ferris, and A. Green. 2001. Fecal shedding of Salmonella spp. by dairy cows on farm and at cull cow markets. J. Food Prot. 64:3-11. https://doi .org/10.4315/0362-028X-64.1.3.

Zankari, E., H. Hasman, S. Cosentino, M. Vestergaard, S. Rasmussen, O. Lund, F. M. Aarestrup, and M. V. Larsen. 2012. Identification of acquired antimicrobial resistance genes. J. Antimicrob. Chemother. 67:2640-2644. https://doi.org/10.1093/jac/dks261.

Zhang, S., H. C. den Bakker, S. Li, J. Chen, B. A. Dinsmore, C. Lane, A. C. Lauer, P. I. Fields, and X. Deng. 2019. SeqSero2: Rapid and improved Salmonella serotype determination using whole-genome sequencing data. Appl. Environ. Microbiol. 85:e01746-19. https:// doi.org/10.1128/AEM.01746-19. 